\title{
The immunological response to syphilis differs by HIV status; a prospective observational cohort study
}

\author{
Chris Kenyon ${ }^{1,2^{*}}$ D, Kara Krista Osbak' ${ }^{1}$ Tania Crucitti $^{3}$ and Luc Kestens ${ }^{4,5}$
}

\begin{abstract}
Background: It is not known if there is a difference in the immune response to syphilis between HIV-infected and uninfected individuals.

Methods: We prospectively recruited all patients with a new diagnosis of syphilis and tested their plasma for IFNa, IFNy, IL-1 $\beta$, IL-12p40, IL-12p70, IP-10, MCP-1, MIP-1a, MIP-1 $\beta$, IL-4, IL-5, IL-6, IL-7, IL-8, IL-10 and IL-17A at baseline pre-treatment and 6 months following therapy.

Results: A total of 79 HIV-infected [44 primary/secondary syphilis (PSS) and 35 latent syphilis (LS)] and 12 HIV-uninfected (10 PSS and 2 LS) cases of syphilis and 30 HIV-infected controls were included in the study. At the baseline visit, compared to the control group, concentrations of IL-10 were significantly elevated in the HIV-infected and uninfected groups. The level of IL-10 was significantly higher in the HIV-infected compared to the HIV-uninfected PSS group (25.3 pg/mL (IQR, 4.56-41.76) vs $2.73 \mathrm{pg} / \mathrm{mL}$ (IQR, 1.55-9.02), P = 0.0192). In the HIV-infected PSS group (but not the HIV-infected LS or HIV-uninfected PSS groups) the IP-10, MIP-1 b, IL-6 and IL-8 were raised compared to the controls. IL-10 levels decreased but did not return to control baseline values by 6 months in HIV infected PSS and LS and HIV uninfected PSS.

Conclusion: PSS and LS in HIV-infected individuals is characterized by an increase in inflammatory and anti-inflammatory cytokines such as IL-10. The increase of IL-10 is greater in HIV-infected than uninfected individuals. Further work is required to ascertain if this is part of an immunological profile that correlates with adverse outcomes such as serofast syphilis and neurosyphilis, in HIV-infected individuals.
\end{abstract}

Keywords: Syphilis, HIV, Immunology, IL-10, Treponema pallidum

\section{Background}

Syphilis is a multi-stage disease caused by infection with Treponema pallidum subsp. pallidum (T. pallidum). A number of studies have found the natural course of syphilis to be altered in HIV-infected patients. The alterations include a higher rate of asymptomatic primary disease [1], more aggressive primary or secondary stage disease $[2,3]$ and a higher proportion (up to a quarter) presenting with mixed primary and secondary stage disease at the time of diagnosis $[3,4]$. There is also evidence that neurosyphilis may occur more frequently, at

\footnotetext{
* Correspondence: ckenyon@itg.be

${ }^{1}$ HIV/STI Unit, Institute of Tropical Medicine, Antwerp, Belgium

${ }^{2}$ Division of Infectious Diseases and HIV Medicine, University of Cape Town,

Anzio Road, Observatory 7700, Cape Town, South Africa

Full list of author information is available at the end of the article
}

an earlier stage and progress more rapidly in the presence of HIV infection [5-7]. Serological cure in the cerebrospinal fluid (CSF) [8] and peripheral blood [3, 9-11] has been shown to be slower in HIV-infected individuals. Unusual serological diagnostic test results such as false negative treponemal and non-treponemal tests in syphilis may also be more common among persons with HIV infection [12, 13].

These findings generate the hypothesis that the immune responses to syphilis differ by HIV-infection status. Most previous studies have characterized the immune response to syphilis in animal models or in HIV-uninfected individuals [14-20]. The only study that we are aware of that has included an HIV-infected population was weakened by its retrospective study design, 
small sample size, small number of cytokines evaluated and lack of HIV-uninfected and healthy HIV-infected control groups [21].

Establishing if there is a differential immune response to T. pallidum infection by HIV status, and more fully characterizing what this response is in HIV-infected individuals, is important for a number of reasons. Firstly, in countries where the incidence of syphilis is increasing, a large proportion of syphilis infections are occurring in HIV-infected persons [22-24]. Typically, an increasing proportion of cases occur in individuals with repeat syphilis $[22,25]$. This repeat syphilis is often asymptomatic and diagnosed purely on the basis of increased titres of non-treponemal tests $[22,26]$. Since a wide array of other factors can cause changes in these titres, a proportion of these diagnoses of repeat syphilis may be false positives [22, 25]. If a sufficiently distinctive immunological profile of initial and repeat syphilis infection could be established, then this could be used in verifying the diagnosis of syphilis in these reinfection cases. Given the fact that in some populations a majority of syphilis reinfections are in HIV-infected individuals, an important first step in this process is determining what the immune response to syphilis is in HIV-infected versusuninfected individuals [22]. Secondly, if immune response to syphilis was found to be subverted in HIV then this would pave the way for studies to investigate if this was causally linked to certain poor outcomes in syphilis, such as early neurosyphilis [7].

\section{Methods}

The Institutional Review Board of the Institute of Tropical Medicine (ITM) and the Ethics Committee of the University Hospital Antwerp approved this substudy (13/44/426). Between May 2014 and June 2015, participants were recruited in the main study "Treponema pallidum-specific Proteomic Changes in Patients With Incident Syphilis Infection (SeTPAT)" study (ClinicalTrials.gov Registration Number: NCT02059525) conducted at the ITM in Antwerp, Belgium. All patients attending the ITM, Antwerp's STI or HIV clinics, over the age of 17 years in whom a new diagnosis of syphilis was made and had not received antibiotics in the preceding 30 days were prospectively recruited into the study (See Additional file 1: Figure S1 for Participant Inclusion Process). The diagnosis and staging of syphilis was according to the Centers for Disease Control and Prevention classification [27]. All patients were assessed according to a standardized protocol that collected detailed information about sexual behavior, clinical features and laboratory tests including Rapid Plasma Reagin test (RPR), Treponema pallidum Particle Agglutination (TPPA) and C-Reactive Protein (CRP). The RPR titre was tested using Macro-Vue RPR card tests (Becton
Dickinson, Sparks, MD, USA) and the TPPA with SERODIA-TPPA (Fujirebio Inc., Tokyo, Japan), according to the manufacturer's instructions. HIV viral loads below the limit of detection (20 copies $/ \mathrm{mL}$ ) were given the value of 10 copies $/ \mathrm{mL}$. The presence of concomitant sexually transmitted infections (STIs) (gonorrhea and chlamydia) was assessed via an Abbott RealTime CT/NG assay (Abbott Molecular, Des Plaines, IL, USA).

Patients were followed up at 3, 6, 9 and 12 months. Patient clinical and laboratory characteristics were recorded at each consultation. In addition, 30 HIV positive controls attending the same HIV clinic and at the same time as the cases were recruited. Blood was drawn into EDTA-coated tubes (Sarstedt Monovette, Nümbrecht, Germany) and separated by centrifugation at $2000 \mathrm{~g}$ for $10 \mathrm{~min}$ at ambient temperature. Within $3 \mathrm{~h}$ of collection, plasma was frozen and stored at $-80{ }^{\circ} \mathrm{C}$ until testing. To avoid collection bias all samples were processed in the same fashion according to the study standard operating procedures.

Chemokines (Monocyte Chemoattractant Protein [MCP]-1,

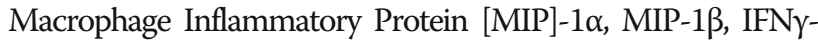
Inducible Protein [IP]-10, Interleukin [IL]-8) and cytokines (Interferon [IFN] $\alpha$, IFN $\gamma$, IL-1 $\beta$, IL-12p40, IL-12p70, IL-4, IL-5, IL-6, IL-7, IL-10 and IL-17A) from the baseline and 6 month post-treatment time-points, and from an additional two individuals 3 and 12 months before their syphilis diagnosis, were measured in a single experiment using a magnetic bead Milliplex ${ }^{\mathrm{m}}$ Human Cytokine kit (Millipore, MA, USA) on a BioPlex $^{\mathrm{Tu}}$ Suspension Array Reader (Bio-Rad Laboratories Inc ${ }^{\oplus}, \mathrm{CA}, \mathrm{USA}$ ) in accordance with the manufacturer's instructions. Standard curves were constructed using duplicate measurements of kit standards. Samples below the limit of quantification were assigned the value of half the lowest limit detected for each cytokine (see Additional file 1: Table S1).

\section{Statistical analyses}

Values are summarized as medians and interquartile ranges (IQR). In keeping with previous studies, we found that the immunological profiles of syphilis differed between primary and secondary stage syphilis combined group (PSS) and early and late latent stage syphilis combined group (LS) $[20,21]$. All results are therefore presented stratified by PSS versus LS. There were only two HIV-uninfected patients diagnosed with LS and no comparisons are therefore made with this group. Comparison of the inter-individual data between the PSS, and LS groups was performed using the Mann-Whitney $U$-test. Wilcoxon signed rank test was used to evaluate intra-individual changes from baseline to six-month visit. Correlations were assessed via Spearman's rank correlation coefficient. Due to the small sample size and 
the fact that this is the first study of its kind, the study was conceived as being exploratory rather than hypothesis testing. This, combined with the fact that the cyto- and chemokines under investigation are known to be inter-dependent, led us to not utilize Bonferroni corrections [28-30]. All analyses were performed in Stata 13 (StataCorp LP, College Station, TX, USA). A $P$-value of less than 0.05 was considered statistically significant.

\section{Results}

A total of 79 HIV-infected [44 PSS and 35 LS] and 12 HIV-uninfected (10 PSS and 2 LS) cases of syphilis and 30 HIV-infected controls were included in the study (see Additional file 1: Figure S1 for inclusion process). At baseline, the age, number of sex partners in the prior 12 months, proportion with a previous episode of treated syphilis, prevalence of other STIs diagnosed at baseline, RPR titre and syphilis treatment received did not differ significantly between HIV-infected and uninfected PSS groups (Table 1). Likewise, except for a higher HIV viral load in the controls than the PSS and LS groups, there was no significant difference in the age, CD4 $\mathrm{T}$ cell count, percent on antiretroviral therapy (ART) or HIV viral load between the controls and HIVinfected syphilis patients. All patients were treated with intramuscular benzathine penicillin G, except for two patients who were treated with oral doxycycline. Only one participant was a woman. She was HIV negative and presented with secondary syphilis. All the men with syphilis, excluding two individuals, reported being men who have sex with men (MSM).

\section{Baseline immunological profile}

At the baseline visit, compared to the control group, concentrations of the anti-inflammatory cytokine IL-10 were significantly elevated in both the HIV-infected and uninfected groups (Fig. 1; Table 2). The level of IL-10 was strikingly higher in the HIV-infected PSS compared to the HIV-uninfected PSS group (25.3 pg/mL (IQR, 4.56-41.76) versus $2.73 \mathrm{pg} / \mathrm{mL}$ (IQR, 1.55-9.02), $P=0.0192$ ). In the HIV-infected PSS group (but not the HIVinfected LS or HIV-uninfected PSS groups) the chemokines IP-10, MIP-1 $\beta$, IL-8 and the pro-inflammatory cytokine IL-6 were raised significantly compared to the controls. Of these only the MIP- $1 \beta$ level was significantly higher in the HIV-infected PSS than uninfected PSS group $(P=0.0205)$.

\section{Six-month immunological profile}

In seven out of 91 individuals with syphilis we had baseline but no 6-month samples. At 6 months post syphilis treatment the levels of IL-10 had declined in all groups, and most strikingly in the HIV-infected PSS group. IL-10 remained significantly elevated compared to the control group $(P<0.0001$ to 0.0018$)$. There was no longer a difference in IL-10 levels between the HIV-infected and uninfected PSS groups.

Table 1 Baseline characteristics

\begin{tabular}{|c|c|c|c|c|c|}
\hline & \multirow{2}{*}{$\begin{array}{l}\text { Controls } \\
(n=30)\end{array}$} & \multicolumn{3}{|c|}{ Primary/Secondary syphilis } & \multirow{2}{*}{$\begin{array}{l}\text { Latent Syphilis } \\
\mathrm{HIV}+(n=35)^{\#}\end{array}$} \\
\hline & & $\mathrm{HIV}+(n=44)^{\#}$ & HIV- $(n=10)^{\#}$ & $H I V+$ Vs. HIV-, $P^{\$}$ & \\
\hline Men & $30(100 \%)$ & $44(100 \%)$ & $9(90 \%)^{*}$ & 0.0359 & $35(100 \%)$ \\
\hline Age (years) & $37(32-45)$ & $39.5(30.5-50.0)$ & $34(30-43)$ & 0.4689 & $40(35-46)$ \\
\hline MSM & $24(80 \%)$ & $44(100 \%)$ & $9(90 \%)$ & 0.0359 & $34(94.7 .1 \%)$ \\
\hline CD4 T cell count (cells per $\mu \mathrm{L}$ ) & $577(392-684)$ & $649(452-838)$ & $800(800-800)$ & 0.4537 & $590(433-691)$ \\
\hline HIV Viral Load (copies per mL) & $34(10-814)$ & $10(10-53)^{*}$ & NA & NA & $10(10-25)^{* *}$ \\
\hline On Antiretroviral Therapy & $24(80 \%)$ & $39(88.6 \%)$ & NA & NA & $31(88.6 \%)$ \\
\hline RPR titre & $0(0-0)$ & 1/64 (1/32-1/128) & $1 / 64(1 / 16-1 / 128)$ & 0.5556 & $1 / 64(1 / 16-1 / 128)$ \\
\hline Treatment & & & & 1.0 & \\
\hline Benzathine-penicillin G & NA & $44(100 \%)$ & $10(100 \%)$ & & $33(94.3 \%)$ \\
\hline Doxycycline & NA & 0 & 0 & & $2(5.7 \%)$ \\
\hline Previous treated syphilis & NA & $27(61.3 \%)$ & $3(30 \%)$ & 0.0743 & $24(68.6 \%)$ \\
\hline Other STIs present ${ }^{a}$ & $0(30)$ & $1(2.3 \%)$ & $0(0 \%)$ & 0.63 & $2(5.7 \%)$ \\
\hline No. of sex partners prior 12 months & $1.5(1-6)$ & $8.5(2-26)^{* *}$ & $6.5(2-8)^{*}$ & 0.5174 & $7(1-12)^{*}$ \\
\hline
\end{tabular}

Data are $\mathrm{n}(\%)$, median (IQR), unless otherwise stated. NA - Not Applicable

$* P<0.05, * * P<0.005$

${ }^{\#} P$-value is for comparison with controls at baseline (Mann-Whitney U-test, excluding 'Men', 'MSM', 'On Antiretroviral Therapy' and 'Other STIs' where Fisher's exact test used)

${ }^{\$} P$-value is for comparison between HIV-infected and uninfected groups (Mann-Whitney U-test, except 'Men', 'MSM', 'Treatment', 'Previous treated syphilis' and 'Other STIs' where Fisher's exact test used)

aPresence of $N$. gonorrhoeae or C. trachomatis in urethra, rectum or oropharynx

MSM: men who have sex with men 

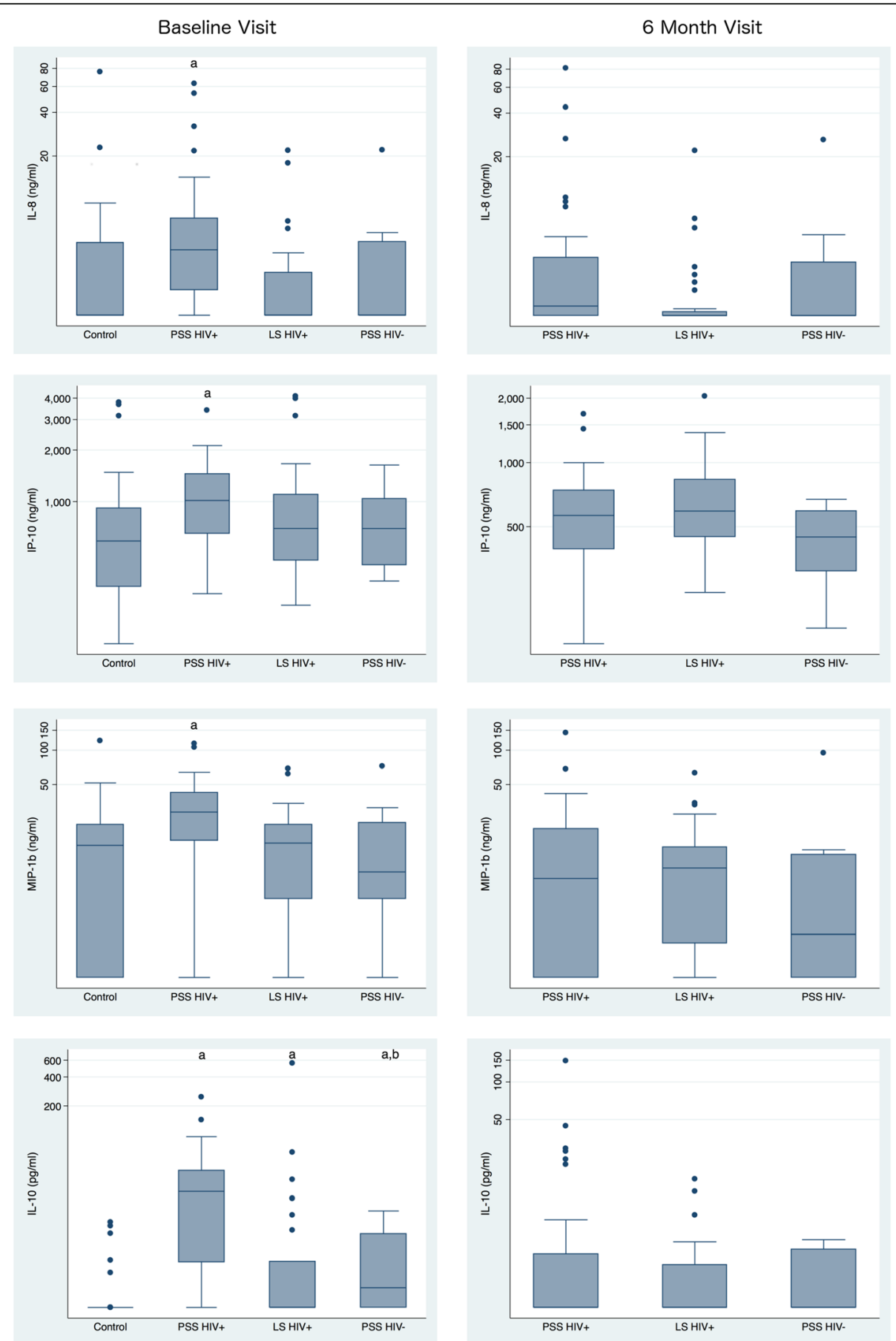

Fig. 1 Plasma cytokine and chemokine concentrations in HIV-infected and uninfected patients with syphilis by syphilis stage at baseline and 6 month visit. Cytokine levels in each group ( $\mathrm{pg} / \mathrm{ml}, \mathrm{Y}$ axis log scale) are shown as median and interquartile range (box), with 10th and 90th percentiles (whiskers). Letters (a) and (b) above the box-plot indicate statistically significant differences $(P<0.05)$ as compared with the HIV-infected controls and the comparison between HIV-infected and uninfected PSS groups, respectively. Abbreviations. PSS HIV+: Primary Secondary Syphilis HIV-infected, LS HIV+: Latent Syphilis HIV-infected, PSS HIV-: Primary Secondary Syphilis HIV-uninfected

Another interesting observation was that IFNa levels declined in all groups and at 6 months attained significantly lower levels than the HIV-infected control group $(P=0.0007$ to 0.0104$)$.

\section{Intra-individual change in IL-10 \& IFNa concentrations}

IL-10

There was a significant decline in IL-10 from baseline to 6 month visit in the HIV-infected PSS group $(P<0.0001)$, 
Table 2 Plasma cytokine and chemokine concentrations in HIV-infected and uninfected patients with syphilis by syphilis stage (pg/ml)

\begin{tabular}{|c|c|c|c|c|c|}
\hline \multirow{3}{*}{ Baseline } & \multirow[t]{2}{*}{ HIV-infected Controls } & \multicolumn{2}{|l|}{ HIV-infected } & \multirow{2}{*}{$\begin{array}{l}\text { HIV-uninfected } \\
\text { Primary/Secondary Syphilis\# }\end{array}$} & \multirow[b]{2}{*}{ PSS HIV+ Vs. - P $^{\$}$} \\
\hline & & Primary/Secondary Syphilis" & Latent Syphilis" & & \\
\hline & & & & & \\
\hline & 30 & 44 & 35 & 10 & \\
\hline \multicolumn{6}{|c|}{ Pro-inflammatory } \\
\hline $\mathrm{IFNa}$ & $9.88(1.6-16.43)$ & $7.45(0.8-31.35)$ & $7.45(0.8-20.42)$ & $4.12(0.8-24.73)$ & 0.5202 \\
\hline $\operatorname{IL} 1 \beta$ & $1.57(1.57-1.57)$ & $1.57(1.57-1.57)$ & $1.57(1.57-1.57)$ & $1.57(1.57-1.57)$ & 0.4883 \\
\hline IL-6 & $1.26(1.26-1.26)$ & $1.26(1.26-1.26)^{*}$ & $1.26(1.26-1.26)$ & $1.26(1.26-1.26)$ & 0.722 \\
\hline IL-17A & $1.52(1.52-1.52)$ & $1.52(1.52-1.52)$ & $1.52(1.52-1.52)$ & $1.52(1.52-1.52)$ & 0.8358 \\
\hline \multicolumn{6}{|l|}{ Th1 } \\
\hline IFNY & $2.18(1.54-4.59)$ & $3.52(1.54-5.45)$ & $1.79(1.54-4.59)$ & $4.01(1.54-16.66)$ & 0.5991 \\
\hline IL-7 & $1.11(1.11-1.11)$ & $1.11(1.11-1.36)$ & $1.11(1.11-1.11)$ & $1.11(1.11-1.11)$ & 0.357 \\
\hline IL-12p40 & $1.02(1.02-1.02)$ & $1.02(1.02-1.02)$ & $1.02(1.02-1.02)$ & $1.02(1.02-1.02)$ & 0.4056 \\
\hline IL-12p70 & $1.61(1.61-1.61)$ & $1.61(1.61-1.67)$ & $1.61(1.61-1.61)$ & $1.61(1.61-4.08)$ & 0.6264 \\
\hline \multicolumn{6}{|l|}{ Chemokines } \\
\hline IL-8 & $1.6(1.6-5.06)$ & $4.51(2.41-7.43)^{* *}$ & $1.6(1.6-3.15)$ & $1.6(1.6-5.13)$ & 0.0723 \\
\hline IP-10 & 589.4 (319.38-917.05) & $1016.1(649.64-1451.32)^{* *}$ & $696.89(455.35-1101.67)$ & $696.52(426.27-1040.07)$ & 0.1561 \\
\hline MCP-1 & $258.42(179.09-307.75)$ & 260.1 (216.95-307.35) & $242.05(180.35-303.1)$ & 247.64 (205.76-287.32) & 0.4321 \\
\hline MIP-1a & $1.63(1.63-3.65)$ & $3.09(1.63-8.3)$ & $1.63(1.63-1.63)$ & $1.63(1.63-1.63)$ & 0.1247 \\
\hline MIP-1 $\beta$ & $14.54(0.99-22.23)$ & $28.59(16.06-42.51)^{* *}$ & $15.2(4.92-22.23)$ & $8.46(4.92-23.05)$ & 0.0205 \\
\hline \multicolumn{6}{|l|}{ Th2 } \\
\hline IL-4 & $1.58(1.58-1.58)$ & $1.58(1.58-1.58)$ & $1.58(1.58-1.58)$ & $1.58(1.58-1.58)$ & 0.4883 \\
\hline IL-5 & $1.58(1.58-1.58)$ & $1.58(1.58-1.58)$ & $1.58(1.58-1.58)$ & $1.58(1.58-1.58)$ & 0.3324 \\
\hline \multicolumn{6}{|c|}{ Anti-inflammatory } \\
\hline IL-10 & $1.55(1.55-1.55)$ & $25.3(4.56-41.76)^{* * *}$ & $2.46(1.55-4.62)^{*}$ & $2.73(1.55-9.02)^{*}$ & 0.0029 \\
\hline \multicolumn{6}{|l|}{6 month } \\
\hline $\mathrm{N}$ & & 41 & 34 & 10 & \\
\hline \multicolumn{6}{|c|}{ Pro-inflammatory } \\
\hline IFNa & & $0.8(0.8-9.27)^{* * \#}$ & $0.8(0.8-7.8)^{* * \#}$ & $0.8(0.8-0.8)^{* \#}$ & 0.3282 \\
\hline $\mathrm{IL}-1 \beta$ & & $1.57(1.57-1.57)$ & $1.57(1.57-1.57)$ & $1.57(1.57-1.57)$ & 0.1798 \\
\hline IL-6 & & $1.26(1.26-1.26)$ & $1.26(1.26-1.26)$ & $1.26(1.26-1.26)$ & 0.7379 \\
\hline IL-17A & & $1.52(1.52-1.52)$ & $1.52(1.52-1.52)$ & $1.52(1.52-1.52)$ & 0.7379 \\
\hline \multicolumn{6}{|l|}{ Th1 } \\
\hline IFNY & & $1.54(1.54-5.09)$ & $1.62(1.54-4.88)$ & $5.17(1.54-13.89)$ & 0.0978 \\
\hline IL-7 & & $1.11(1.11-1.11)$ & $1.11(1.11-1.11)$ & $1.11(1.11-1.11)$ & 0.6486 \\
\hline IL-12p40 & & $1.02(1.02-1.02)$ & $1.02(1.02-1.02)$ & $1.02(1.02-1.02)$ & 0.2068 \\
\hline IL-12p70 & & $1.61(1.61-1.61)$ & $1.61(1.61-1.86)$ & $1.61(1.61-1.61)$ & 0.7639 \\
\hline \multicolumn{6}{|l|}{ Chemokines } \\
\hline IL-8 & & $1.87(1.6-4.02)$ & $1.6(1.6-1.7)$ & $1.66(1.6-3.75)$ & 0.4406 \\
\hline IP-10 & & $564.56(392.18-740.03)$ & $592.04(447.8-831.96)$ & 447 (309-593) & 0.2134 \\
\hline MCP-1 & & $270.25(225.5-330.3)$ & $261.32(241.06-312.26)$ & 269 (219-297) & 0.8287 \\
\hline MIP-1a & & $1.63(1.63-1.63)$ & $1.63(1.63-1.63)$ & $1.63(1.63-1.63)$ & 0.6726 \\
\hline MIP-1 $\beta$ & & $7.4(0.99-20.28)$ & $9.17(1.99-14)$ & $2.39(0.99-12.05)$ & 0.249 \\
\hline
\end{tabular}


Table 2 Plasma cytokine and chemokine concentrations in HIV-infected and uninfected patients with syphilis by syphilis stage (pg/ml) (Continued)

\begin{tabular}{lllll}
\hline Th2 & & & & \\
IL-4 & $1.58(1.58-1.58)$ & $1.58(1.58-1.58)$ & $1.58(1.58-1.58)$ & 0.1798 \\
IL-5 & $1.58(1.58-1.58)$ & $1.58(1.58-1.58)$ & $1.58(1.58-1.58)$ & 0.3621 \\
Anti-inflammatory & & & & \\
IL-10 & $1.83(1.83-4.09)^{* * *}$ & $2.11(1.83-3.37)^{* * *}$ & $2.26(1.83-4.46)^{* *}$ & 0.6296 \\
\hline
\end{tabular}

All values are median and interquartile range. Data are $\mathrm{n}(\%)$, median (IQR), unless otherwise stated

\# $P$-value is for comparison with controls at baseline (Mann-Whitney $U$-test)

${ }^{\$} P$-value is for comparison between HIV-infected and uninfected groups with Primary/Secondary syphilis (Mann-Whitney $U$-test)

${ }^{*} P<0.05$, ** $P<0.005$, *** $P<0.0005$

PSS - Primary/Secondary Syphilis

and a non-significant decline in the HIV-infected LS group and the HIV-uninfected PSS group $(P=0.4803$ and 0.4838 , respectively; Table 2 \& Fig. 2).

\section{IFNa}

Over the same time period, there was a significant decline in IFN $\alpha$ in the HIV-infected LS group $(P<$ $0.0412)$, but this change was borderline significant in the HIV-infected PSS group $(P=0.0568)$ and not significant in the HIV-uninfected PSS group $(P=0.3173$; Table 2 \& Fig. 1).

In the two individuals for which we were able to test their cytokine profile before, during and after PSS infection there was an increase in IL-10 at the time of infection and a return to pre-syphilis values after 6 months. These individuals were originally included as controls and then during the course of the study developed a syphilis infection. Conversely, IFNa levels in these two decreased at the time of secondary stage syphilis diagnosis and continued to drop until the 6-month visit.

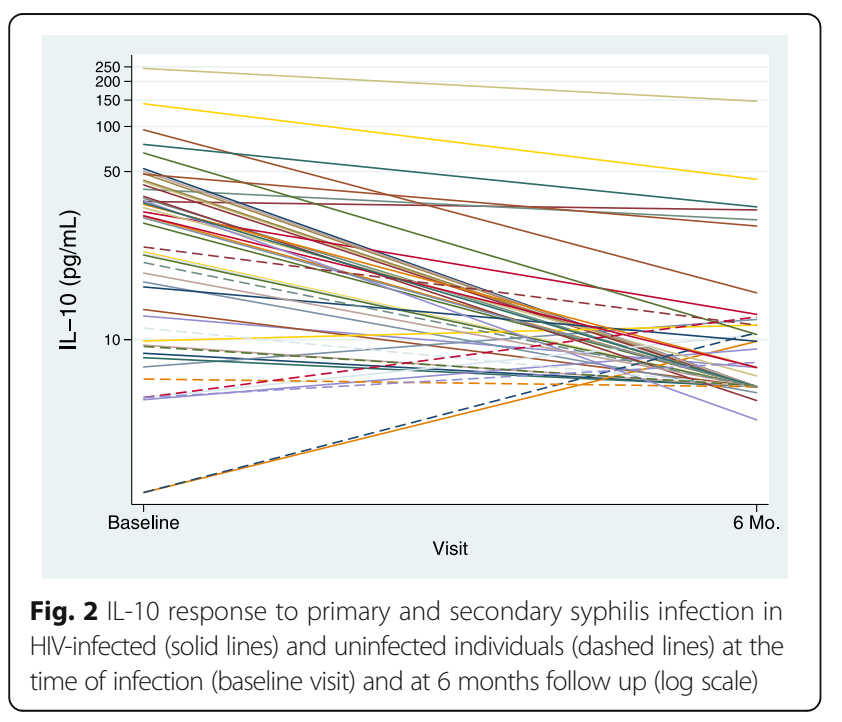

\section{IL-10 Correlation analyses}

At the baseline visit there were differences between the HIV-infected and uninfected groups in the immunological markers that were correlated with IL-10 level. In the HIV-infected PSS group, IL-10 was significantly positively correlated with IL-12p40, IL-12p70, MIP-1 $\alpha$, MIP-1 $\beta$, IL-5, IL-6, IL-7 and IL-8 $(\mathrm{r}=0.36,0.67,0.59$, $0.61,0.75,0.79,0.83,0.53$, respectively; Additional file 1: Table S2a). In the HIV-uninfected group there were no significant correlations with IL-10 (Additional file 1: Table S2b). There were however only 10 individuals in this group.

None of the cohort was diagnosed with treatment failure or neurosyphilis subsequent to the baseline visit and we were thus unable to assess if any particular immunological profile was predictive of these adverse outcomes.

In sensitivity analyses we excluded the one woman from the analyses and repeated the analyses with the PSS group split into primary syphilis (PS) and secondary syphilis (SS) and the LS group split into early latent and late latent syphilis. We also repeated the analyses with the HIV-infected group limited to those taking ART. These made little difference to the results (results not shown). Scatterplots revealed that the difference in IL-6 between controls and the HIV-infected PSS group was largely driven by two outlier values. Repeating the comparison between these two groups excluding these two outliers, there was no longer a statistically higher IL-6 level in the HIV-infected group.

\section{Discussion}

The most prominent difference in the plasma immunological response to PSS between the HIV-infected and uninfected was a ten-fold higher IL-10 response in the HIV positive group. Although this is a new finding, it is not incommensurate with findings from other studies. The study by Knudsen et al. [21] found similarly high levels of IL-10 in HIV-infected PSS $(46.7 \mathrm{pg} / \mathrm{mL}$; IQR 28.4-78.9) and LS (13.4 pg/mL; IQR 6.5-31.1). Many studies in HIV-uninfected populations have not assessed IL-10 levels [15, 17, 31]. Those that have, found either 
IL-10 to be not raised $[14,19]$ or marginally raised [16, 20] compared to HIV-uninfected controls. These studies used differing methodologies and as a result IL-10 values cannot be meaningfully compared between studies. Our study builds on these results by for the first time comparing immunological profiles in HIV-infected and uninfected groups.

\section{How should we interpret the higher IL-10 levels in the} HIV-infected individuals and in the PSS group in particular? The initial immune response to T. pallidum involves a robust cell mediated response characterized by a Th1 predominance that results in the clearance of the vast majority of treponemes at the lesion of the primary stage $[15,32]$. We mainly found increased levels of IL-10 and chemokines in the PSS group. The positive correlations we found between IL-10 and IL-12p40, IL-12p70, MIP$1 \alpha$, MIP-1 $\beta$, IL-5, IL-6, IL-7 and IL- 8 in the HIVinfected PSS group could be interpreted as evidence that the increased IL-10 is acting as a feedback response to limit inflammation. We did not see any particular increase of Th1 cytokines as reported by two other studies $[15,29]$.

An alternative/complementary explanation is that $T$. pallidum is playing a role in the IL-10 elevation. In a proportion of cases, T. pallidum is able to evade the immune system and set up secondary and chronic latent stages of infection [14]. Poor antigenicity and antigenic variation have been shown to play an important role in this regard [33-35]. A further mechanism is that an enhanced regulatory T-cell (Treg) response in early syphilis may down-regulate the immune response to T. pallidum and thereby facilitate its survival $[17,31,36]$. IL-10 plays an important role in the establishment of the Treg antiinflammatory effect that is important in preventing overwhelming inflammatory responses [37]. These immunosuppressive properties of IL-10 have also been shown to be harnessed by a number of pathogens to facilitate persistent infection: Borrelia burgdorferi [38], Plasmodium spp. [39], Leishmania spp. [40] and Mycobacterium tuberculosis [37].

Babolin et al. provided experimental evidence that the T. pallidum protein, TpF1 bacterioferrin, is able to induce a Treg response in patients with secondary syphilis via the induction of IL-10 and TGF- $\beta$ [41]. Podwinska and colleagues investigated the ability of lymphocytes to produce cytokines from patients at different stages of syphilis in response to stimulation with T. pallidum antigen [20]. They noted a stepwise increase in IL-10 production from primary syphilis to secondary and latent syphilis that correlated inversely with the ability to produce IL-2 (a Th1 cytokine). In a study of 531 syphilis patients with and without neurosyphilis, $\mathrm{Li}$ and colleagues found evidence that Tregs play a role in treponemal persistence and the genesis of neurosyphilis in HIVuninfected individuals [17]. They demonstrated that secondary and serofast syphilis patients had increased Treg percentages in their peripheral blood compared with healthy controls. Patients with neurosyphilis had a higher frequency of Tregs in peripheral blood compared to nonneurosyphilis patients. In a similar vein, Pastuszczak et al., found that IL-10 was considerably higher in the CSF of patients with neurosyphilis than syphilis patients without neurological involvement [19]. These findings are also consistent with the clinical study by Knudsen et al., which was able to demonstrate with a high degree of probability that $T$. pallidum infection was the cause of the IL-10 elevation [21].

HIV-infection is characterized by a Th1 to Th2 cytokine shift during the course of the infection [42] which includes an enhanced potential for robust IL-10 and associated Treg response [43-45]. If HIV infection leads to a more pronounced Treg response to infection with T. pallidum, then this could explain a number of the atypical features of syphilis in HIV-infected individuals mentioned in the introduction. The recently established correlation between Treg activity and risk of serofast syphilis and neurosyphilis [17] in HIV-uninfected individuals suggests the need for more investigations along these lines in HIV co-infected patients. The discovery of an immunological profile strongly associated with asymptomatic neurosyphilis would be of considerable clinical utility.

Limitations in our study include that we only measured systemic cytokine responses and not cytokine secretions in response to antigenic (T. pallidum antigen) stimulation. The detection of cytokines in plasma samples has several limitations. First of all, cytokine responses are mainly local and short-ranged. We only detect cytokine overflow in the systemic circulation. Secondly, we have no data about the cell types that secreted these and conclusions are thus largely speculative. Thirdly, cytokine measurements in plasma are critically dependent on proper sample collection, storage and the technology used to detect them. We ensured that the cytokine detection was done under optimal circumstances such as prompt sample processing in order to minimize undesirable test variation due to suboptimal storage and test conditions.

In addition, the small sample size and the fact that almost the entire cohort were MSM, with high-risk behavior, thereby reducing the generalizability of the results. We did not resample the control group at 6-months and thus did not have a control group for the 6-month samples. The fact that those diagnosed with syphilis reported a higher number of sexual partners than the controls raises the possibility that they were co-infected with other undiagnosed STIs. Given the fact that there was 
no statistically significant difference in the number of partners, or the diagnosis of STIs between the PSS HIVinfected and uninfected subjects, this explanation is unlikely to explain the immunological differences found between these two groups. Our HIV-infected patients included both those receiving and not receiving ART, which may have influenced our results, however, we consider this as unlikely as repeating the analyses restricted to those on ART did not substantively change the results. We did not assess patients' genotypes for IL-10 and other cytokines. A number of IL-10 single nucleotide polymorphisms have been found to be associated with both IL-10 inducibility [19] and susceptibility to infectious diseases such as tuberculosis [46] and neurosyphilis [19]. For a number of the chemo- and cytokines, only a small proportion of samples had levels above the level of detection (Additional file 1: Table S1), particularly in the cases of IL1b, IL-6, IL-17A, IL-7, IL12p40, IL-4 and IL-5. A number of these low abundance chemo- and cytokines had low correlations between technical duplicates (Additional file 1: Table S1). The results for these chemo- and cytokines need to be interpreted with considerable caution. Furthermore, we did not correct for multiple comparisons which may have led to type I errors [28]. The strengths of the study include its setting within a prospective observational cohort study wherein the patients had their behavioral, clinical and laboratory characteristics collected in a detailed and standardized fashion.

\section{Conclusion}

In conclusion, we demonstrate that PSS and LS in HIV-infected individuals is characterized by not only an increase in inflammatory- but also the anti-inflammatorycytokine, IL-10. The increase of IL-10 is greater in HIVinfected than uninfected individuals and declines but does not return to baseline levels by 6 -month post- infection. Further work is required to ascertain if there is an immunological profile that specifically correlates with adverse outcomes such as treatment failure, serofast- and neurosyphilis in HIV-co-infected individuals.

\section{Additional file}

Additional file 1: Table S1. Cytokine data quality assessment. Table S2.a Correlations between cytokines/chemokines in primary/secondary syphilis in HIV-infected individuals at baseline. Table S2.b Correlations between cytokines/chemokines in primary/secondary syphilis in HIV-uninfected individuals at baseline. (DOCX $51 \mathrm{~kb}$ )

\section{Abbreviations}

ART: Antiretroviral therapy; CRP: C-Reactive Protein; CSF: Cerebrospinal fluid; HIV: Human Immunodeficiency Virus; IL: Interleukin; IQR: Interquartile range; ITM: Institute of Tropical Medicine; LS: Latent Syphilis; MCP: Monocyte Chemoattractant Protein; MIP: Macrophage Inflammatory Protein; MSM: Men who have sex with men; PS: Primary Syphilis; PSS: Primary/Secondary Syphilis;
RPR: Rapid Plasma Reagin test; SS: Secondary Syphilis; STI: Sexually Transmitted Infection; TPPA: Treponema pallidum Particle Agglutination

\section{Acknowledgements}

We would like to thank Said Abdellati and Vicky Cuylaerts for performing the cytokine assays.

\section{Funding}

This work was supported by the grants from the Flanders Research

Foundation, SOFI-B Grant to CK, http://www.fwo.be.

\section{Availability of data and materials}

The datasets generated during the current study are not publicly available due to privacy but are available from the corresponding author on reasonable request.

\section{Authors' contributions}

CK conceptualized the study. CK was responsible for the acquisition, analysis and interpretation of data. CK, KO, TC and LK played a role in writing, editing and approving the final version.

\section{Competing interests}

The authors declare that they have no competing interests.

\section{Consent for publication}

Not applicable.

\section{Ethics approval and consent to participate}

This study was approved by the Institutional Review Board of the Institute of Tropical Medicine (ITM) and the Ethics Committee of the University Hospital Antwerp approved this study (13/44/426). Written informed consent was obtained from each participant.

\section{Author details}

${ }^{1}$ HIV/STI Unit, Institute of Tropical Medicine, Antwerp, Belgium. ${ }^{2}$ Division of Infectious Diseases and HIV Medicine, University of Cape Town, Anzio Road, Observatory 7700, Cape Town, South Africa. ${ }^{3}$ HIV/STI Reference Laboratory, Institute of Tropical Medicine, Antwerp, Belgium. ${ }^{4}$ Immunology Unit, Institute of Tropical Medicine "Antwerp, Belgium. ${ }^{5}$ Department of Biomedical

Sciences, University of Antwerp, Antwerp, Belgium.

Received: 7 October 2016 Accepted: 11 January 2017

Published online: 31 January 2017

\section{References}

1. Karumudi UR, Augenbraun M. Syphilis and HIV: a dangerous duo. Expert Rev Anti Infect Ther. 2005;3(5):825-31.

2. Kassutto S, Doweiko JP. Syphilis in the HIV era. Emerg Infect Dis. 2004;10(8): 1471-3.

3. Schofer $H$, Imhof M, Thoma-Greber E, Brockmeyer NH, Hartmann M, Gerken G, Pees HW, Rasokat H, Hartmann H, Sadri l, et al. Active syphilis in HIV infection: a multicentre retrospective survey. The German AIDS Study Group (GASG). Genitourin Med. 1996;72(3):176-81.

4. Hutchinson CM, Hook 3rd EW, Shepherd M, Verley J, Rompalo AM. Altered clinical presentation of early syphilis in patients with human immunodeficiency virus infection. Ann Intern Med. 1994;121(2):94-100.

5. Flood JM, Weinstock HS, Guroy ME, Bayne L, Simon RP, Bolan G. Neurosyphilis during the AIDS epidemic, San Francisco, 1985-1992. J Infect Dis. 1998;177(4): 931-40.

6. Centers for Disease Control Prevention. Symptomatic early neurosyphilis among HIV-positive men who have sex with men-four cities, United States, January 2002-June 2004. MMWR Morb Mortal Wkly Rep. 2007;56(25):625.

7. Tsuboi M, Nishijima T, Teruya K, Kikuchi Y, Gatanaga H, Oka S. Cerebral Syphilitic Gumma within 5 Months of Syphilis in HIV-Infected Patient. Emerg Infect Dis. 2016;22(10):1846-8.

8. Marra CM, Maxwell CL, Tantalo L, Eaton M, Rompalo AM, Raines C, Stoner BP, Corbett JJ, Augenbraun M, Zajackowski M, et al. Normalization of cerebrospinal fluid abnormalities after neurosyphilis therapy: does HIV status matter? Clin Infect Dis. 2004;38(7):1001-6. 
9. Ghanem KG, Erbelding EJ, Wiener ZS, Rompalo AM. Serological response to syphilis treatment in HIV-positive and HIV-negative patients attending sexually transmitted diseases clinics. Sex Transm Infect. 2007:83(2):97-101.

10. Malone JL, Wallace MR, Hendrick BB, LaRocco Jr A, Tonon E, Brodine SK, Bowler WA, Lavin BS, Hawkins RE, Oldfield 3rd EC. Syphilis and neurosyphilis in a human immunodeficiency virus type-1 seropositive population: evidence for frequent serologic relapse after therapy. Am J Med. 1995;99(1):55-63.

11. Rolfs RT, Joesoef MR, Hendershot EF, Rompalo AM, Augenbraun MH, Chiu M, Bolan G, Johnson SC, French P, Steen E, et al. A randomized trial of enhanced therapy for early syphilis in patients with and without human immunodeficiency virus infection. The Syphilis and HIV Study Group. N Engl J Med. 1997; 337(5):307-14

12. Jurado RL, Campbell J, Martin PD. Prozone phenomenon in secondary syphilis. Has its time arrived? Arch Intern Med. 1993;153(21):2496-8.

13. Smith G, Holman RP. The prozone phenomenon with syphilis and HIV-1 co-infection. South Med J. 2004;97(4):379-82.

14. Cruz AR, Ramirez LG, Zuluaga AV, Pillay A, Abreu C, Valencia CA, La Vake C, Cervantes JL, Dunham-Ems S, Cartun R, et al. Immune evasion and recognition of the syphilis spirochete in blood and skin of secondary syphilis patients: two immunologically distinct compartments. PLoS Negl Trop Dis. 2012;6(7):e1717.

15. Engelkens HJ, ten Kate FJ, Judanarso J, Vuzevski VD, van Lier JB, Godschalk JC van der Sluis JJ, Stolz E. The localisation of treponemes and characterisation of the inflammatory infiltrate in skin biopsies from patients with primary or secondary syphilis, or early infectious yaws. Genitourin Med. 1993;69(2):102-7.

16. Kojima N, Bristow CC, Maecker H, Rosenberg-Hasson Y, Leon SR, Vargas SK, Konda KA, Caceres CF, Klausner JD. Similarities in the Markers of Inflammation Between Men With Syphilis and Women With Increased Risk of HIV Acquisition. Clin Infect Dis. 2016;62(2):265-6.

17. Li K, Wang C, Lu H, Gu X, Guan Z, Zhou P. Regulatory T cells in peripheral blood and cerebrospinal fluid of syphilis patients with and without neurological involvement. PLoS Negl Trop Dis. 2013;7(11):e2528.

18. Pastuszczak M, Gozdzialska A, Jakiela B, Obtulowicz A, Jaskiewicz J, Wojas-Pelc A. Robust pro-inflammatory immune response is associated with serological cure in patients with syphilis: an observational study. Sex Transm Infect. 2017;93(1):11-4. doi:10.1136/sextrans-2016-052681. Epub 2016 Jun 29.

19. Pastuszczak M, Jakiela B, Jaworek AK, Wypasek E, Zeman J, Wojas-Pelc A. Association of Interleukin-10 promoter polymorphisms with neurosyphilis. Hum Immunol. 2015;76(7):469-72.

20. Podwinska J, Lusiak M, Zaba R, Bowszyc J. The pattern and level of cytokines secreted by Th1 and Th2 lymphocytes of syphilitic patients correlate to the progression of the disease. FEMS Immunol Med Microbiol. 2000;28(1):1-14.

21. Knudsen A, Benfield T, Kofoed K. Cytokine expression during syphilis infection in HIV-1-infected individuals. Sex Transm Dis. 2009:36(5):300-4.

22. Kenyon C, Lynen L, Florence E, Caluwaerts S, Vandenbruaene M, Apers L, Soentjens $P$, Van Esbroeck M, Bottieau E. Syphilis reinfections pose problems for syphilis diagnosis in Antwerp, Belgium-1992 to 2012. Eurosurveillance. 2014;19(45):22-9.

23. European Centre for Disease Prevention and Control. Sexually transmitted infections in Europe, 1990-2010. Stockholm: ECDC; 2012.

24. Read P, Fairley CK, Chow EP. Increasing trends of syphilis among men who have sex with men in high income countries. Sex Health. 2015;12(2):155-63.

25. Brewer TH, Peterman TA, Newman DR, Schmitt K. Reinfections During the Florida Syphilis Epidemic, 2000-2008. Sex Transm Dis. 2011;38(1):12-7.

26. Kerani R, Lukehart S, Stenger M, Marra C, Pedersen R, Golden M. Is early latent syphilis more likely in patients with a prior syphilis infection? London: British Society for Sexual Health and HIV, Presentation at: 18th International Society for STD Research; 2009.

27. Workowski KA, Berman SM. Centers for Disease C, Prevention: Sexually transmitted diseases treatment guidelines, 2010: Department of Health and Human Services, Centers for Disease Control and Prevention. 2010.

28. Perneger TV. What's wrong with Bonferroni adjustments. BMJ. 1998;316(7139): 1236-8.

29. Armstrong RA. When to use the Bonferroni correction. Ophthalmic Physiol Opt. 2014;34(5):502-8.

30. Rothman KJ. No adjustments are needed for multiple comparisons. Epidemiology. 1990;1(1):43-6.

31. Fitzgerald TJ. The Th1/Th2-like switch in syphilitic infection: is it detrimental? Infect Immun. 1992;60(9):3475-9.

32. Van Voorhis WC, Barrett LK, Koelle DM, Nasio JM, Plummer FA, Lukehart SA. Primary and secondary syphilis lesions contain mRNA for Th1 cytokines. J Infect Dis. 1996;173(2):491-5.
33. Radolf JD, Norgard MV, Schulz WW. Outer membrane ultrastructure explains the limited antigenicity of virulent Treponema pallidum. Proc Natl Acad Sci U S A. 1989;86(6):2051-5.

34. LaFond RE, Molini BJ, Van Voorhis WC, Lukehart SA. Antigenic variation of TprK $V$ regions abrogates specific antibody binding in syphilis. Infect Immun. 2006;74(11):6244-51.

35. LaFond RE, Centurion-Lara A, Godornes C, Van Voorhis WC, Lukehart SA. Tprk sequence diversity accumulates during infection of rabbits with Treponema pallidum subsp. pallidum Nichols strain. Infect Immun. 2006; 74(3):1896-906,

36. Tabor DR, Kiel DP, Jacobs RF. Cyclophosphamide-sensitive activity of suppressor T cells during treponemal infection. Immunology. 1987;62(1):127-32.

37. Redford PS, Murray PJ, O'Garra A. The role of IL-10 in immune regulation during M. tuberculosis infection. Mucosal Immunol. 2011;4(3):261-70.

38. Giambartolomei GH, Dennis VA, Philipp MT. Borrelia burgdorferi stimulates the production of interleukin-10 in peripheral blood mononuclear cells from uninfected humans and rhesus monkeys. Infect Immun. 1998;66(6):2691-7.

39. Medina TS, Costa SP, Oliveira MD, Ventura AM, Souza JM, Gomes TF, Vallinoto AC, Povoa MM, Silva JS, Cunha MG. Increased interleukin-10 and interferon-gamma levels in Plasmodium vivax malaria suggest a reciprocal regulation which is not altered by IL-10 gene promoter polymorphism. Malar J. 2011;10:264

40. Murphy ML, Wille U, Villegas EN, Hunter CA, Farrell JP. IL-10 mediates susceptibility to Leishmania donovani infection. Eur J Immunol. 2001;31(10):2848-56.

41. Babolin C, Amedei A, Ozolins D, Zilevica A, D'Elios MM, de Bernard M. TpF1 from Treponema pallidum activates inflammasome and promotes the development of regulatory T cells. J Immunol. 2011;187(3):1377-84.

42. Klein SA, Dobmeyer JM, Dobmeyer TS, Pape M, Ottmann OG, Helm EB, Hoelzer D, Rossol R. Demonstration of the Th1 to Th2 cytokine shift during the course of HIV-1 infection using cytoplasmic cytokine detection on single cell level by flow cytometry. AIDS. 1997;11(9):1111-8.

43. Orsilles MA, Pieri E, Cooke P, Caula C. IL-2 and IL-10 serum levels in HIV-1infected patients with or without active antiretroviral therapy. APMIS. 2006;114(1):55-60

44. Srikanth P, Castillo RC, Sridharan G, John TJ, Zachariah A, Mathai D, Schwartz DH. Increase in plasma IL-10 levels and rapid loss of CD4+ T cells among HIV-infected individuals in south India. Int J STD AIDS. 2000;11(1):49-51.

45. Li JC, Lee DC, Cheung BK, Lau AS. Mechanisms for HIV Tat upregulation of IL-10 and other cytokine expression: kinase signaling and PKR-mediated immune response. FEBS Lett. 2005;579(14):3055-62.

46. Meenakshi P, Ramya S, Shruthi T, Lavanya J, Mohammed HH, Mohammed SA, Vijayalakshmi V, Sumanlatha G. Association of IL-1 beta +3954 C/T and IL-10-1082 G/A cytokine gene polymorphisms with susceptibility to tuberculosis. Scand J Immunol. 2013;78(1):92-7.

\section{Submit your next manuscript to BioMed Central and we will help you at every step:}

- We accept pre-submission inquiries

- Our selector tool helps you to find the most relevant journal

- We provide round the clock customer support

- Convenient online submission

- Thorough peer review

- Inclusion in PubMed and all major indexing services

- Maximum visibility for your research

Submit your manuscript at www.biomedcentral.com/submit 